\title{
Nutrición parenteral domiciliaria en pacientes pediátricos con insuficiencia intestinal
}

\section{Home parenteral nutrition in pediatric patients with intestinal insufficiency}

\author{
Yazmín Zapata Olivaresa, María Isabel Hodgson Bunster, ${ }^{\mathrm{a}, \mathrm{b}}$, María Luisa Cordero Bayón', \\ Lorena Rodríguez Osiac ${ }^{\mathrm{d}}$, Jaime Cerda Lorca ${ }^{\mathrm{e}}$, Grupo Chileno para el estudio \\ de la Nutrición Parenteral Prolongada en Pediatría
}

\author{
aDepartamento de Nutrición, Diabetes y Metabolismo, Escuela de Medicina, Pontificia Universidad Católica de Chile. Santiago, Chile \\ bepartamento de Gastroenterología y Nutrición, División de Pediatría, Escuela de Medicina, Pontificia Universidad Católica de Chile. Santiago, Chile \\ cPrograma de Nutrición Infantil, Servicio de Pediatría, Hospital Sótero del Río. Santiago, Chile \\ dDepartamento de Nutrición y Alimentos, Ministerio de Salud, Chile \\ eDepartamento de Salud Pública, Facultad de Medicina, Pontificia Universidad Católica de Chile. Santiago, Chile
}

Recibido el 28 de junio de 2018; aceptado el 25 de octubre de 2018

\section{Resumen}

Introducción: La Nutrición Parenteral Domiciliaria (NPD) ha demostrado ofrecer importantes beneficios para los pacientes y el sistema de salud. En Chile se desconoce el número de pacientes que se encuentran recibiendo esta modalidad de tratamiento o que podrían ser candidatos a ella. Objetivo: Determinar la prevalencia y características clínicas de pacientes con Insuficiencia Intestinal (II) que reciben actualmente NPD o que son candidatos a esta. Pacientes y Método: Estudio descriptivo transversal que incluyó pacientes de 0 a 18 años, con diagnóstico de II que se encontraban recibiendo NP por un tiempo superior a 3 meses en el domicilio, o en el hospital con situación clínica estable y con catéter venoso de larga duración (CVC). A través de una encuesta digitalizada se recolectaron y estudiaron las variables: sexo, antecedentes de nacimiento, indicación para iniciar soporte nutricional parenteral, edad de inicio de NP, tipo de CVC utilizado, frecuencia de NP, estado nutricional, alimentación al último control y complicaciones asociadas al uso de NP. Se utilizó el Programa SPSS Statistics, Versión 21, Macintosh, para el análisis de los datos. El análisis descriptivo consideró análisis de frecuencia y medidas de tendencia central. La prueba de Chi cuadrado y de Fisher se usaron para la comparación de proporciones. Resultados: Se registraron datos de 46 pacientes, cuya edad promedio fue de 55,5 meses. La principal indicación para iniciar la Nutrición Parenteral (NP) fue la disminución de la superficie intestinal (78,3\%.). El 63\% de los pacientes se encontraba hospitalizado. No se encontraron diferencias significativas entre el lugar de tratamiento y las variables estado nutricional e infecciones de catéter en el último año. Conclusiones: Se identificó la prevalencia de pacientes con NP prolongada y sus características clínicas. No se encontraron diferencias que respalden la entrega de este tratamiento en el hospital por sobre el domicilio. Es necesario desarrollar políticas públicas que garanticen la opción de recibir este tratamiento en el domicilio.
Palabras clave: Nutrición parenteral prolongada; nutrición parenteral domiciliaria en pediatría; insuficiencia intestinal; registro 


\begin{abstract}
Introduction: Home parenteral nutrition (HPN) has been shown to offer important benefits for patients and the health system. In Chile, the number of patients who are receiving this type of treatment or who could be candidates for it is unknown. Objective: To determine the prevalence and clinical characteristics of patients with intestinal insufficiency (II) currently receiving HPN or who are candidates for it. Patients and Method: Cross-sectional descriptive study which included patients aged between 0 to 18 years with diagnosis of II who were receiving parenteral nutrition (PN) for over three months, either at home or in the hospital, with a stable clinical situation and a long-term venous catheter (CVC). Through a digitalized survey, the following variables were collected and studied: gender, birth history, indication to initiate parenteral nutritional support, age of initiation of PN, type of CVC, frequency of PN, nutritional status and feeding in the last control and complications associated with the use of PN. Data analysis was performed using the SPSS Statistics Software, Version 21, Macintosh. The descriptive analysis considered frequency analysis and central trend measures. The Chi-square and Fisher tests were used for comparison of proportions. Results: Data from 46 patients were recorded. The average age was 55.5 months. The main indication for initiating the PN was the decrease of the intestinal surface $(78.3 \%) .63 \%$ of the patients were hospitalized. No significant differences were found between the place of treatment and the nutritional status and catheter infections variables in the last year. Conclusions: The prevalence of patients with long-term PN and their clinical characteristics were identified. No differences were found to support the administration of this treatment in the hospital over the home. Public policies must be developed to guarantee the option of receiving this treatment at home.
\end{abstract}

\section{Keywords:}

Long-term parenteral nutrition; pediatric home parenteral nutrition; intestinal insufficiency; registry

\section{Introducción}

La Nutrición Parenteral (NP) constituye el pilar fundamental en la terapia de variadas patologías, pudiendo ser indicada por periodos agudos o prolongados. Su objetivo, en pediatría, es entregar el aporte nutricional necesario para lograr un crecimiento óptimo, cuando la alimentación oral o enteral no es posible, o es insuficiente. Su uso ha permitido la supervivencia de pacientes que antes eran considerados "fuera del alcance terapéutico"1.

La Insuficiencia Intestinal (II), es un buen ejemplo de cómo el Soporte Nutricional Prolongado es parte esencial del tratamiento que estos niños reciben. Se la define como la incapacidad de alcanzar un adecuado balance proteico, energético, hídrico, electrolítico y de micronutrientes debido a una reducción de la masa funcional enterocitaria, impidiendo una adecuada absorción y digestión de nutrientes, ya sea producto de reducciones anatómicas de la longitud intestinal, patologías neuromusculares del tracto gastrointestinal o alteraciones del epitelio intestinal ${ }^{1,2}$.

Si bien este tipo de enfermedades son poco frecuentes, son de alto impacto emocional, social y económico para el paciente y su familia, requiriendo de atención especializada y multidisciplinaria, lo que muchas veces se traduce en largas estadías hospitalarias.

Por esto, la Nutrición Parenteral Domiciliaria (NPD) representa para este tipo de pacientes, una alternativa a la hospitalización prolongada, $\mathrm{y}$ ha sido re- conocida como la mejor opción para mejorar la calidad de vida de estos niños y sus familias ${ }^{3}$. Los beneficios de la asistencia en domicilio comparados con el tratamiento hospitalario, han sido descritos acabadamente desde hace ya un par de décadas. Entre ellos cabe destacar: i) Beneficios socio-emocionales: permitiendo la inserción familiar y escolar del niño, además la integración familiar; ii) Beneficios clínicos: representados en un menor número de complicaciones infecciosas; iii) Beneficios de gestión de recursos sanitarios: en donde es posible evidenciar una reducción del costo asociado al tratamiento, llegando a un ahorro de casi el $75 \%{ }^{4-9}$.

Si bien en Chile existen instituciones que prestan esta opción de tratamiento, esto no se realiza de manera universal ni requiere ser notificado, por lo que se desconoce cuantos pacientes se encuentran actualmente con NPD o son candidatos a esta modalidad de tratamiento, estando posiblemente hospitalizados por largos periodos.

Debido a esto, se hace indispensable conocer el número de pacientes candidatos a este tipo de tratamiento, lo cual permitiría implementar políticas públicas y contar a futuro con un programa nacional de NPD.

El objetivo de este estudio fue conocer la prevalencia y describir el perfil clínico y demográfico de pacientes en edad pediátrica, con diagnóstico de II que se encontrasen recibiendo NP prolongada como parte de su tratamiento. 


\section{Pacientes y Método}

Estudio descriptivo transversal que incluye datos de hospitales de alta complejidad pertenecientes a la Red Pública Asistencial de Salud y a la Red Privada de Salud UC Christus. Las instituciones fueron contactadas por vía telefónica y/o correo electrónico a través de los Jefes de Servicio.

Se incluyeron pacientes entre 0 y 18 años de edad, con dependencia de NP (total o parcial) por un tiempo superior a tres meses, que estuvieran en el domicilio u hospitalizados en condiciones de alta domiciliaria, definido como: Situación clínica estable y con Catéter Venoso Central (CVC) de larga duración in situ.

Los datos fueron recolectados desde Julio de 2016 a Julio de 2017, mediante un instrumento digitalizado de 23 preguntas, basado en la encuesta "Sustain Baseline Data Collection Form, ASPEN 2014”, que debían llenar los médicos tratantes. Las variables estudiadas fueron: sexo, datos de nacimiento, diagnóstico, indicación para iniciar soporte nutricional parenteral, edad de inicio de NP, tipo de catéter venoso utilizado, frecuencia de NP, estado nutricional al último control, alimentación al último control y complicaciones asociadas al uso de NP.

Para la evaluación e interpretación de los datos antropométricos se utilizó la puntuación z. Los parámetros considerados fueron: peso para la talla $(\mathrm{P} / \mathrm{T})$ y talla para la edad (T/E) en los menores de 5 años, los cuales fueron calculados mediante el programa estadístico WHO Anthro (versión 2.1.1, enero 2011). En los mayores de 5 años, se consideró la puntuación $\mathrm{z}$ de los indicadores Índice de Masa Corporal para la edad (IMC/E), y Talla para la edad (T/E), los cuales fueron calculados con el programa estadístico WHO Anthro Plus. Se realizó el análisis conjunto de los valores z, utilizando los criterios de clasificación del estado nutricional propuestos por la OMS. En pacientes con menos de 32 semanas de gestación al momento del na-

Tabla 1. Antecedentes Neonatales del total de pacientes $(n=46)$

\begin{tabular}{lcc}
\hline & $\begin{array}{c}\text { Frecuencia } \\
(\mathrm{N})\end{array}$ & $\begin{array}{c}\text { Porcentaje } \\
(\%)\end{array}$ \\
\hline Edad gestacional al nacimiento (semanas) & 5 & \\
$\quad<32$ & 20 & 10,9 \\
$32-36$ & 21 & 43,5 \\
$>36$ & & 45,7 \\
Clasificación Peso de Nacimiento & 16 & 34,8 \\
PEG & 29 & 63,0 \\
AEG & 1 & 2 \\
GEG & & \\
\hline
\end{tabular}

PEG: Pequeño para la edad gestacional (PN <p10 para la EG); AEG: adecuado para la edad gestacional (PN entre p10-p90 para la EG); GEG: grande para la edad gestacional (PN >p90 para la EG). cimiento se corrigió la edad cronológica hasta los 24 meses de edad, y en aquellos pacientes que nacieron entre las 32 y 36 semanas de gestación, se corrigió la edad cronológica hasta los 12 meses de edad, según las fórmulas establecidas por el Ministerio de Salud de Chile ${ }^{10,11}$.

Para la descripción de costos de día cama promedio se utilizaron datos publicados por el Fondo Nacional de Salud (FONASA) en Agosto de 2017.

Para el análisis de relación entre las variables infección de catéter en el último año y estado nutricional según lugar de tratamiento, se consideraron sólo aquellos pacientes que llevasen un tiempo superior a 6 meses en el hospital o domicilio según cada caso.

Se utilizó el Programa SPSS Statistics, Versión 21 para Macintosh para el análisis de los datos. El análisis descriptivo consideró análisis de frecuencia y medidas de tendencia central. La prueba de Chi cuadrado y de Fisher se usaron para la comparación de proporciones.

\section{Consideraciones éticas}

Se obtuvo la aprobación del comité de ética de la Facultad de Medicina de la Pontificia Universidad Católica de Chile, otorgando una dispensa de consentimiento informado para el retiro de los datos, además de la aprobación de la Subsecretaría de Redes Asistenciales y el Departamento de Nutrición y Alimentos del Ministerio de Salud de Chile.

\section{Resultados}

\section{Antecedentes neonatales y clínicos}

Se contactaron 27 hospitales públicos de alta complejidad que contaban con un Servicio de Pediatría de referencia regional. De estos, el $48,2 \%(n=13)$ contaba con uno o más pacientes que cumplían los criterios de inclusión. Del total de centros participantes, el 53,8\% $(n=7)$ se encuentran ubicados en la región metropolitana y el porcentaje restante corresponde a hospitales emplazados en otras regiones del país.

Se incluyeron 46 pacientes en la base de datos. El $84,8 \%(\mathrm{n}=39)$ provenía de alguna institución pública del país y el $15,2 \%(n=7)$ restante provenía de una institución privada. Del total de pacientes, el 56,5\% era de sexo femenino $(\mathrm{n}=26)$. La edad promedio al momento del estudio fue de 55,5 meses ( $\pm 48,8 \mathrm{DE}$ ), con un mínimo de 6 meses y un máximo de 18 años de edad, encontrándose el 52\% $(\mathrm{n}=24)$ en el grupo etario de los preescolares (de 2 a 5 años de edad).

En cuanto a los antecedentes neonatales, un 10,9\% $(\mathrm{n}=5)$ de los pacientes nació antes de las 32 semanas de edad gestacional $(\mathrm{EG})$, un $4,3 \%(\mathrm{n}=2)$ tuvo un peso de nacimiento (PN) inferior a los 1000 grs, y un $34,8 \%(\mathrm{n}=16)$ fue Pequeño para la edad gestacional (PEG) (tabla 1). 
El 71,7\% de los pacientes $(n=33)$ comenzó el soporte nutricional parenteral antes del primer mes de vida. La principal indicación para esto fue la disminución de superficie intestinal en un 78,3\% de los casos, el porcentaje restante correspondió a patologías causantes de alteración de la motilidad intestinal o afectación extensa del epitelio intestinal (tabla 2).

El diagnóstico con mayor prevalencia fue Gastrosquisis en un $32,7 \%$, pudiendo estar o no asociada a vólvulo o atresia intestinal.

Las características del intestino remanente se describen en la tabla 2.

\section{Lugar de tratamiento, días de hospitalización y posibilidad de asistencia a la educación formal}

El 63\% de los pacientes $(n=29)$ se encontraba hospitalizado al momento del estudio, el $37 \%$ restante estaba recibiendo su tratamiento en el domicilio, el cual era administrado por un profesional de enfermería de una empresa externa o por los padres previamente instruidos en el manejo. De los pacientes que se encontraban en domicilio $(\mathrm{n}=17)$, siete eran atendidos en el sistema privado de salud.

El tiempo promedio de soporte parenteral, independiente del lugar de tratamiento, era de 1365 días (DE $\pm 1.272,3$ días) al momento del estudio. El promedio de días de hospitalización de aquellos niños que se encontraban recibiendo tratamiento en el hospital era de 791 días ( $\mathrm{DE} \pm$ 579,7; min: 102, máx: 2.374 días), y 927 días (DE \pm 815,8; min: 166, máx: 3.025 días) de espera en el hospital, previa al alta domiciliaria, para aquellos pacientes que estaban en el domicilio.

El $48 \%$ de los pacientes que se encontraban en el hospital eran preescolares, en el grupo de tratamiento domiciliario este rango etario alcanzó el 58,8\%.

En relación a la incorporación al sistema educativo de aquellos pacientes en edad preescolar o escolar $(\mathrm{n}=34)$ el $47,1 \%(\mathrm{n}=16)$ no estaba inserto en ningún tipo de actividad escolar, considerando como tal, asistencia a jardín infantil o colegio, escuela hospitalaria o estimulación por parte de programas del centro de referencia. De ellos, el 56,3\% $(\mathrm{n}=9)$ se encontraba hospitalizado.

\section{Alimentación y estado nutricional actual}

El 71,7\% ( $\mathrm{n}=33)$ de los pacientes se encontraba recibiendo NP diariamente, y un $19,9 \%$ con una frecuencia de 4 o menos días a la semana.

La mayoría de los niños se encontraba recibiendo NP más alimentación mixta y suplementación con fórmula polimérica $(n=21)$ o elemental $(n=9)$, el detalle de esto se presenta en la tabla 3.

En aquellos pacientes con pérdidas gastrointestinales importantes $(\mathrm{n}=7)$, se realizó reposición de fluidos diariamente en 4 de ellos $(57,1 \%)$, el porcentaje restante lo necesitó menos de tres veces por semana.
Para el análisis de estado nutricional actual, se consideraron 45 pacientes del total; ya que un paciente (lactante) presentaba Desnutrición Marásmica Severa (Indicador P/T -6,02 DE) debido a un agotamiento de accesos venosos; lo que sugiere que se suspendió la NP por periodos prolongados, por lo que fue excluido del análisis global y solo se menciona en forma aislada.

El $62,2 \%$ de los pacientes presenta un estado nutricional adecuado para su estatura. Sin embargo, el $53,3 \%$ de los pacientes posee una talla por debajo de lo normal, con una media para la variable $\mathrm{z} T / \mathrm{E}$ de $-2,16$ $( \pm 1,78 \mathrm{DE})($ tabla 4$)$.

Tabla 2. Características clínicas y diagnósticos del total de pacientes $(n=46)$

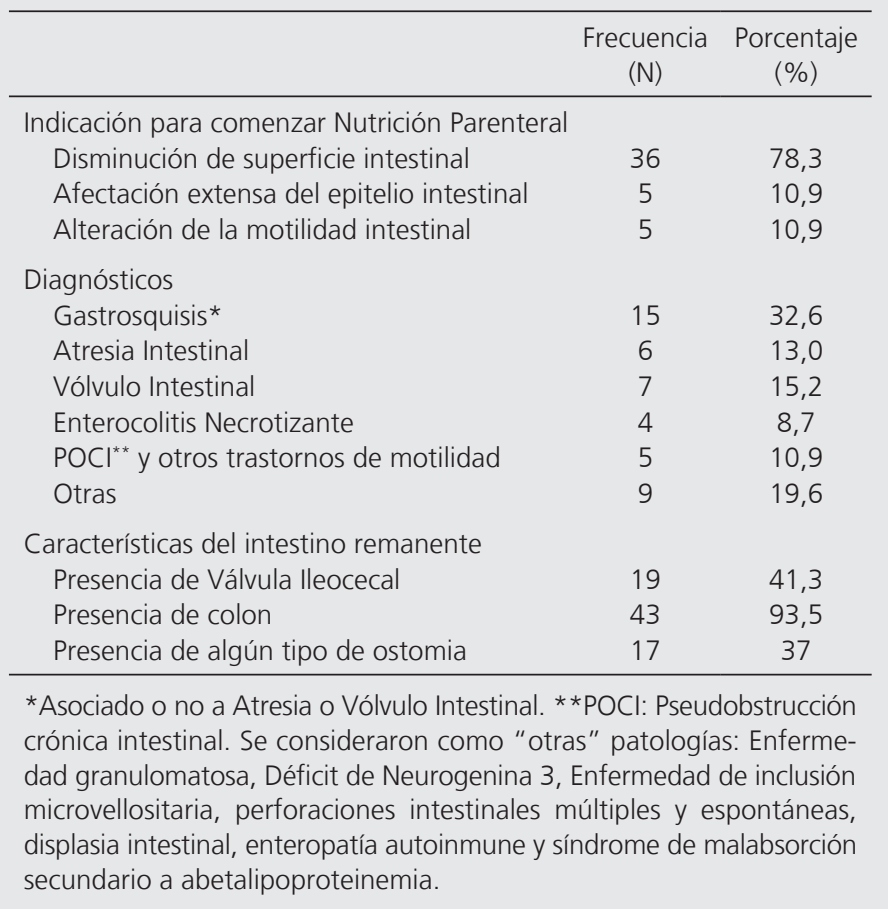

Tabla 3. Frecuencia de Tipo de Alimentación al último control

\begin{tabular}{lcc}
\hline Tipo de Alimentación & $\begin{array}{c}\text { Frecuencia } \\
(\mathrm{N})\end{array}$ & $\begin{array}{c}\text { Porcentaje } \\
(\%)\end{array}$ \\
\hline NP Exclusiva & 4 & 8,7 \\
NP + Estímulo enteral* & 5 & 10,9 \\
NP + Formula enteral & 7 & 15,2 \\
NP + Alimentación mixta + Fórmula & 30 & 65,2 \\
\hline
\end{tabular}

NP: Nutrición Parenteral. *Estímulo enteral se definió como $10-20 \mathrm{ml} / \mathrm{kg} / \mathrm{d}$ con leche materna, fórmula elemental o polimérica. 
Tabla 4. Estado nutricional al último control $(n=45)$

\begin{tabular}{|c|c|c|c|}
\hline & Frecuencia (N) & & centaje (\%) \\
\hline \multicolumn{4}{|l|}{ Estado nutricional } \\
\hline Desnutrición & 3 & & 6,7 \\
\hline Riesgo de desnutrir & 7 & & 15,6 \\
\hline Eutrofia & 28 & & 62,2 \\
\hline Sobrepeso & 7 & & 15,6 \\
\hline \multicolumn{4}{|l|}{ Talla } \\
\hline Talla baja & 24 & & 53,3 \\
\hline Talla normal & 20 & & 44,4 \\
\hline Talla alta & 1 & & 2,2 \\
\hline \multicolumn{4}{|c|}{ Indicadores del Estado nutricional al último control $(n=45)$} \\
\hline Z score & $\mathrm{N}$ & Media & DE \\
\hline T/E al último control & 45 & $-2,16$ & $\pm 1,78$ \\
\hline $\mathrm{P} / \mathrm{T}$ al último control & 32 & $-0,28$ & $\pm 1,19$ \\
\hline z-IMC al último control & 13 & $-0,33$ & $\pm 0,95$ \\
\hline
\end{tabular}

T/E: talla/edad. P/T: peso/talla. IMC: Índice de Masa Corporal

\section{Catéter, infecciones y otras complicaciones asociadas}

Un 87\% $(n=40)$ de los pacientes contaban con un catéter tunelizado para la NP, el $13 \%$ restante se en- contraba utilizando catéter central de inserción periférica $(\mathrm{n}=4)$ o Port-a-Cath $(\mathrm{n}=2)$. El 78,3\% $(\mathrm{n}=36)$ presentó al menos una infección de catéter en el último año, de los cuales el 63,9\% $(n=23)$ se encontraba hospitalizado.

En relación a las complicaciones asociadas al uso de nutrición parenteral prolongada, un 50\% de los pacientes presentó colestasia o alteración de pruebas hepáticas de manera transitoria, según informaron sus médicos tratantes. Un 43,5\% $(\mathrm{n}=20)$ señaló haber tenido otras complicaciones, siendo la trombosis de vías venosas la mencionada con mayor frecuencia $(n=5)$, seguida del déficit de vitamina $\mathrm{D}$, ruptura o autoretiro del CVC, colelitiasis y extravasación de vías venosas.

\section{Relación entre el lugar de tratamiento, estado nutricional actual e infecciones de catéter}

No se encontraron diferencias significativas en cuanto a antecedentes neonatales o clínicos entre los pacientes hospitalizados versus aquellos que estaban en el domicilio (tabla 5).

Tampoco se encontraron diferencias significativas en relación al estado nutricional, e infecciones de catéter en el último año según el lugar de tratamiento (tabla 6).

Tabla 5. Características clínicas y neonatales según lugar de tratamiento $(n=39)$ *

\begin{tabular}{|c|c|c|c|c|c|}
\hline & \multicolumn{5}{|c|}{ Lugar de tratamiento } \\
\hline & \multicolumn{2}{|c|}{ Hospital } & \multicolumn{2}{|c|}{ Domicilio } & \multirow[t]{2}{*}{ Valor $\mathrm{p}$} \\
\hline & Frecuencia (N) & Porcentaje (\%) & Frecuencia (N) & Porcentaje (\%) & \\
\hline \multicolumn{6}{|l|}{ Sexo } \\
\hline Hombre & 9 & 34,6 & 5 & 38,5 & \multirow{2}{*}{1,000} \\
\hline Mujer & 17 & 65,4 & 8 & 61,5 & \\
\hline \multicolumn{6}{|l|}{ Semanas de gestación } \\
\hline$<32$ & 4 & 15,4 & 1 & 7,7 & \multirow{2}{*}{0,640} \\
\hline$>32$ & 22 & 84,6 & 12 & 92,3 & \\
\hline \multicolumn{6}{|l|}{ Peso de Nacimiento /Edad gestacional } \\
\hline PEG & 10 & 38,5 & 5 & 38,5 & \multirow{2}{*}{1,000} \\
\hline AEG & 16 & 61,5 & 8 & 61,5 & \\
\hline \multicolumn{6}{|l|}{ Indicación de NP } \\
\hline Disminución superficie intestinal & 21 & 80,8 & 10 & 76,9 & \multirow{3}{*}{0,944} \\
\hline Afectación extensa del epitelio intestinal & 3 & 11,5 & 2 & 15,4 & \\
\hline Alteración motilidad intestinal & 2 & 7,7 & 1 & 7,7 & \\
\hline \multicolumn{6}{|l|}{ Región de tratamiento } \\
\hline Metropolitana & 13 & 50 & 10 & 76 & \multirow[t]{2}{*}{0,107} \\
\hline Otras Regiones & 13 & 50 & 3 & 23,1 & \\
\hline
\end{tabular}

*Para el análisis de estas variables se incluyó sólo a aquellos pacientes que llevasen 6 meses o más en un respectivo lugar de tratamiento (hospital o domicilio). PEG: Pequeño para la edad gestacional (PN <p10 para la EG); AEG: adecuado para la edad gestacional (PN entre p10p90 para la EG). 
Tabla 6. Infección de catéter en el último año $(n=39) *$ y Estado Nutricional según lugar de tratamiento $(n=38)$ *

\begin{tabular}{|c|c|c|c|c|c|}
\hline & \multicolumn{5}{|c|}{ Lugar de tratamiento } \\
\hline & \multicolumn{2}{|c|}{ Hospital } & \multicolumn{2}{|c|}{ Domicilio } & \multirow[t]{2}{*}{ Valor $p$} \\
\hline & Frecuencia (N) & Porcentaje (\%) & Frecuencia (N) & Porcentaje (\%) & \\
\hline \multicolumn{6}{|l|}{ Infección de catéter } \\
\hline No & 6 & 23,1 & 3 & 23,1 & \multirow[b]{2}{*}{1,000} \\
\hline Sí & 20 & 76,6 & 10 & 76,9 & \\
\hline \multicolumn{6}{|l|}{ Estado nutricional } \\
\hline Malnutrición por déficit & 4 & 16 & 2 & 15,4 & \multirow{3}{*}{0,597} \\
\hline Eutrofia & 16 & 64 & 10 & 76,9 & \\
\hline Malnutrición por exceso & 5 & 20 & 1 & 7,7 & \\
\hline
\end{tabular}

\section{Discusión}

El uso de Nutrición Parenteral Prologada es una práctica que ha permitido extender la supervivencia de pacientes antes considerados fuera del alcance terapéutico. Sin embargo, no basta con aumentar en número de años la expectativa de vida, si no se asegura la calidad de ésta. Es así como la indicación de Nutrición Parenteral en el Domicilio surge como una alternativa valiosa a la hora de buscar ambos objetivos.

La organización del soporte nutricional domiciliario varía según el país donde se implementa. España, al igual que Canadá, cuenta con un registro único a nivel nacional (Registro NADYA-SENPE) donde se entrega información de pacientes adultos y pediátricos con NPD. En Francia, el estado provee los fondos necesarios a un número limitado de centros especializados. Sin embargo, en otros países como Estados Unidos, no existe centralización y cada institución tiene la libertad de crear programas de Nutrición Domiciliaria si lo considera pertinente para la población que atiende. En Latinoamérica, Argentina centraliza la entrega de este tratamiento en un único hospital ${ }^{12-18}$.

En Chile, no existen organismos definidos que se encarguen de proporcionar esta modalidad de atención. La NPD tampoco se incluye dentro de las prestaciones de salud que garantiza el estado o los organismos privados. Por esta razón, la posibilidad de salir al domicilio a recibir tratamiento es más bien desigual y depende de las gestiones propias de los familiares, equipos de salud y hospitales.

Este estudio permitió identificar a 46 pacientes, de 13 hospitales públicos y 1 institución privada, que se encontraban recibiendo NP prolongada en el domicilio, o que estando hospitalizados pudiesen ser candidatos a esta modalidad de tratamiento. $\mathrm{Al}$ igual que en otros reportes la disminución de la superficie intestinal es, en más de dos tercios de los pacientes, la principal motivación para iniciar el soporte parenteral. Siendo la Gastrosquisis, Atresia y Vólvulo Intestinal las patologías de base más frecuentes. Esto podría relacionarse con el aumento de diagnósticos de Gastrosquisis en nuestro país en los últimos años ${ }^{19}$. El grupo etario que concentró el mayor número de pacientes fue el de los preescolares, este resultado es diferente a lo descrito en otras cohortes internacionales, por ejemplo la France$\mathrm{sa}$, en donde los pacientes se concentran en el grupo de menores de un año de edad ${ }^{20,21}$. Es importante mencionar, que esta diferencia en la distribución de los grupos etarios podría deberse a que en países desarrollados el trasplante intestinal es una opción de tratamiento para la insuficiencia intestinal ${ }^{22,23}$.

En cuanto a los pacientes en edad preescolar o escolar, cerca de la mitad no se encontraba inserto en ningún tipo de actividad escolar. Del total de pacientes que no estaban escolarizados, sobre el $50 \%$ se encontraba actualmente en condiciones hospitalarias. Este hecho podría ser perjudicial para su estimulación, desarrollo cognitivo y capacidad de desarrollo social.

Todos los pacientes presentaban una situación clínica estable al momento del estudio, la mayoría de ellos tenía un estado nutricional adecuado. Llama la atención el compromiso de talla que se identificó en el $53,3 \%$ de los pacientes, independiente del lugar de tratamiento. Esto podría deberse a un aporte inadecuado de nutrientes en las etapas iniciales de la patología o en momentos de crecimiento acelerado, o bien podría ser el resultado de suspensiones transitorias del soporte nutricional debido a pérdidas de acceso venoso por períodos variables en el transcurso de la enfermedad ${ }^{24}$.

Más de la mitad de los pacientes se encontraba recibiendo NP en el hospital. El promedio de hospitalización es cercano a los dos años (792 $\pm 579,7$ días) en aquellos niños que estaban hospitalizados y dos años 
y medio (927 $\pm 815,8$ días) de espera hospitalaria para aquellos pacientes que ya estaban en el domicilio.

En Chile, según datos emitidos por FONASA el año 2017, el valor diario de hospitalización en un Servicio de Pediatría en un Hospital Público de Alta complejidad, alcanza los $\$ 36.360$ pesos (55,67 USD aprox.), esto sin considerar el soporte parenteral (que tiene un costo variable en cada paciente) ni la eventual hospitalización en una cama de una Unidad Crítica Pediátrica (UPC) en caso de complicaciones. Tomando en cuenta sólo el día cama, el promedio de gasto de los pacientes hospitalizados podría alcanzar cifras cercanas a los $\$ 28.797 .120$ pesos, e incluso superar los cien millones de pesos si se considera la estadía máxima de uno de los pacientes registrados. Los distintos reportes son claros al señalar, que independiente de la modalidad de salud del país, es posible alcanzar un ahorro que supera el $60 \%$ de los recursos en salud, al capacitar a la familia y trasladar al niño a su domicilio. Esto, sin estimar el costo indirecto que significa tener a un hijo hospitalizado, como los traslados, licencias médicas, pérdida de fuerza laboral, etc. ${ }^{5,9}$. Sobre todo considerando que del total de pacientes hospitalizados, el 23,9\% requirió traslado desde su ciudad natal al centro de tratamiento.

Por otro lado, probablemente uno de los mayores temores de los equipos tratantes, y posibles factores limitantes a la hora de plantear la opción de tratamiento en el domicilio son el deterioro del estado nutricional y las infecciones recurrentes de catéter, que podrían eventualmente, significar la pérdida de accesos venosos. Por esta razón, se evaluó si existían diferencias entre estas variables (Estado Nutricional/Infecciones de catéter en el último año) y el lugar de tratamiento de los pacientes. En cuanto al estado nutricional, la mayoría de los pacientes, independiente del lugar de tratamiento, tiene un estado nutricional adecuado. Cabe destacar que gran parte de los pacientes diagnosticados con malnutrición por exceso se encuentran hospitalizados. Esto podría explicarse por dos razones: la creencia en los equipos tratantes que es mejor mantener a estos pacientes con un estado nutricional por sobre la normalidad en caso que pierdan algún acceso venoso; o por la falta de actividad física que conllevan las hospitalizaciones prolongadas. Sin embargo, estos pacientes no tendrían por que considerarse exentos del riesgo que conlleva la obesidad infantil, al igual que en otros niños con sobrepeso u obesidad hospitalizados ${ }^{25}$.

En cuanto a la presencia de infecciones de catéter en el último año, los resultados son idénticos en ambos grupos; esto hace suponer que en este grupo de pacientes, el riesgo de infección es similar en el hospital y en el domicilio. Hubiese sido valioso evaluar si esta infección significó o no la pérdida del acceso venoso ya que en variadas ocasiones, y dependiendo de la experiencia de los equipos de salud, es posible controlar la infección sin necesidad de retirar el catéter ${ }^{26}$.

Desde el punto de vista clínico, no se encontró evidencia significativa que respalde la administración de este tratamiento en el hospital por sobre el domicilio.

El número de pacientes registrados puede parecer pequeño, sin embargo se debe considerar que este evento clínico es una situación poco frecuente. Pese a lo anterior, este estudio incluye un número importante de pacientes comparado con los resultados de otras publicaciones $^{12-18}$.

\section{Conclusiones}

Luego de una exhaustiva recolección y análisis de datos, que incluyó la participación de 12 instituciones de salud de nuestro país, se logró identificar a 46 pacientes pediátricos con II que reciben NP prolongada. La mayoría de ellos se encuentra recibiendo NP en el hospital.

Los resultados de este estudio, no muestran diferencias significativas en las variables infección de catéter en el último año y estado nutricional según el lugar de tratamiento. Por lo que estas variables no debiesen ser limitantes a la hora de considerar esta opción de tratamiento.

Evaluar la posibilidad de entregar la NP en el domicilio, se enmarca en los Derechos del Niño Hospitalizado $^{27}$ y es una práctica exitosa que se realiza desde los años setenta en Europa y Estados Unidos y desde 1985 en Argentina. La NPD ha demostrado, con amplia solvencia científica, ser la mejor opción para mejorar la calidad de vida de estos pacientes y sus familias, además de contribuir a una disminución del gasto asociado al tratamiento.

Por lo expuesto anteriormente, la entrega del soporte parenteral en el domicilio parece ser factible desde el punto de vista clínico. Sin embargo, es necesario desarrollar un registro único nacional de pacientes con Insuficiencia Intestinal, que permita conocer la incidencia de estos casos y tener acceso actualizado a su situación clínica. Se debe también, evaluar la implementación de un programa nacional que permita asegurar los recursos materiales, humanos y de capacitación sanitaria, para poder ofrecer de manera segura y universal el soporte nutricional parenteral en el domicilio.

\section{Responsabilidades éticas}

Protección de personas y animales: Los autores declaran que los procedimientos seguidos se conformaron a las normas éticas del comité de experimentación humana responsable y de acuerdo con la Asociación Médica Mundial y la Declaración de Helsinki. 
Confidencialidad de los datos: Los autores declaran que han seguido los protocolos de su centro de trabajo sobre la publicación de datos de pacientes.

\section{Derecho a la Privacidad y Consentimiento Informa-} do: Los autores declaran que la información ha sido obtenida de datos previos en forma anonimizada, por lo cual el Comité de Ética de Investigación en uso de sus facultades, ha eximido de la obtención de un consentimiento informado, lo cual consta en el acta respectiva.

\section{Conflicto de intereses}

Los autores declaran no tener conflicto de intereses.

\section{Colaboradores}

Grupo Chileno Para el Estudio de la Nutrición Parenteral en Pediatría: Gigliola Alberti Reus, Rosa María Antilef, Paulina Balboa, Carolina Cors, Graciela De la Fuente, Jessica Delgado, Ana Fritis, Mercedes Guevara, Catalina Le Roy, Aída Maggi, Francisco Moraga, Beatriz Morales, Alexa Puchi, María Jesús Rebollo, K. Walewska Schilling, Marcia Teitelboim, Mario Vildo- so, Rossana Villalobos, J. Carlos Villalobos, Carolina Wong.

\section{Agradecimientos}

Dra. Gisela Alarcón Rojas, Subsecretaria de Redes Asistenciales, Ministerio de Salud, Chile. Directores de Hospital, Jefes de Servicios, Pediatras y Nutriólogos Tratantes de los siguientes centros y hospitales: Hospital Roberto del Río (SSMN, Región Metropolitana); Hospital San Juan de Dios (SSMOC, Región Metropolitana); Hospital Félix Bulnes (SSMOC, Región Metropolitana); Hospital San Borja Arriarán (SSMC, Región Metropolitana); Hospital Luis Calvo Mackenna (SSMO, Región Metropolitana); Hospital Exequiel González Cortés (SSMS, Región Metropolitana); Hospital Padre Hurtado (SSSO, Región Metropolitana); Hospital Clínico Pontificia Universidad Católica de Chile (Región Metropolitana).Hospital Carlos Van Buren (SS Valparaíso San Antonio, Región de Valparaíso); Hospital Dr. Gustavo Fricke (SS Viña del Mar Quillota, Región de Valparaíso); Hospital Regional de Rancagua (SS O'Higgins, Región de O'Higgins); Hospital Regional de Talca (SS del Maule, Región del Maule); Hospital Clínico Regional Dr. Guillermo Brant Benavente (SS Concepción, Región de Biobío); Hospital Dr. Hernán Henríquez Aravena (SS Araucanía Sur, Región de la Araucanía).

\section{Referencias}

1. O'Keefe SJD, Buchman AL, Fishbein TM, Jeejeebhoy KN, Jeppesen PB, Shaffer J. Short bowel syndrome and intestinal failure: Consensus definitions and overview. Clin Gastroenterol Hepatol 2006;4 (1):6-10.

2. Alberti G, Le Roy C, Cofré C, Pattillo JC, Domínguez B, Guerra C, et al. Actualización en el manejo de pacientes con insuficiencia intestinal. Rev Chil Pediatr 2014;85 (2):148-146.

3. ESPGHAN. Home Parenteral Nutrition. J Pediatr Gastroenterol Nutr 2005; 41:70-5.

4. Culine S, Chambrier C, Tadmouri A, et al. Home parenteral nutrition improves quality of life and nutritional status in patients with cancer: A French observational multicentre study. Support Care Cancer 2014; 22(7):1867-74.

5. Gottrand F, Staszewski P, Colomb V, Loras-Duclaux I, Guimber D, Marinier E, et al. Satisfaction in different life domains in children receiving home parenteral nutrition and their families. J Pediatr 2005;146(6):793-7.

6. Melville CAS, Bisset WM, Long S, Milla PJ. Counting the cost: Hospital versus home central venous catheter survival.

J Hosp Infect 1997; 35(3):197-205.

7. Colomb V, Fabeiro M, Dabbas M, Goulet O, Merckx J. Central venous catheter-related infections in children on long-term home parenteral nutrition: incidence and risk factors. Clin Nutr 2000; 19(5):355-9.

8. Marshall J, MD MSc; Gadowsky S, MsAC and DA. Economic Analysis of Home vs Hospital-Based Parenteral Nutrition in Ontario, Canada. J Parenter Enter Nutr 2005;29(4):266-9.

9. Winkler M, Smith C. Clinical, Social, and Economic Impacts of Home Parenteral Nutrition Dependence in Short Bowel Syndrome. J Parenter Enter Nutr 2014;38(1):32S-37S.

10. Engle WA. Age terminology during the perinatal period. Pediatrics 2004;114(5): 1362-4.

11. Ministerio de Salud de Chile; Norma Técnica para la supervisión de niños y niñas de 0 a 9 años en la Atención Primaria de Salud, Programa Nacional de Salud de la Infancia. En: Strain H, Moncada JL, Editores. Santiago, Chile; 2014. p. 215-29.
12. Russo T, Arendt B, Teterina A, et al. Changes in Home Parenteral Nutrition Practice Based on the Canadian Home Parenteral Nutrition Patient Registry. J Parenter Enter Nutr 2017;41(5):830-6.

13. Wanden-Berghe C, Villarés J, Moreno Compés M, et al. Nutrición Parenteral Domiciliaria en España 2011 y 2012; informe del grupo de nutrición artificial domiciliaria y ambulatoria NADYA. Nutr Hosp 2014;29(6):1360-5.

14. Fabeiro M, Dalieri M, Martínez M, et al. Nutrición parenteral domiciliaria (NPD): Factibilidad de implementación desde un hospital público. Nutr Hosp 2011;26(6):1435-9.

15. Moreno JM, Shaffer J, Staun M, et al. Survey on legislation and funding of Home Artificial Nutrition in different european countries. Clin Nutr 2001; 20(2):117-23.

16. Mundi MS, Pattinson A, McMahon MT, Davidson J, Hurt RT. Prevalence of Home Parenteral and Enteral Nutrition in the United States. Nutr Clin Pract 2017; 23(6):799-805.

17. Fernandes G, Kaila B, Jeejeebhoy KN; Canadian Home Parenteral Nutrition (HPN) Registry: Validarion and Patient 
outcomes. J Parenter Enter Nutr 2012;36(4): 407-14.

18. Wanden-Berghe $\mathrm{C}$, Campos $\mathrm{C}$, Cuerda C, Gómez C, et al. Nutrición Parenteral Domiciliaria en España durante 2015; informe del grupo de nutrición artificial domiciliaria y ambulatoria NADYA. Nutr Hosp 2016;33(6):1487-90.

19. Nazer J, Karachon L, Cifuentes L, Assar R; Gastroschisis: A pandemic with increasing rates? ECLAMC experiencia in Chile 1982-2015. Rev Chil Pediatr 2016; 87(5):380-6.

20. Colomb V, Dabbas M, Goulet O, et al; Long- term outcome of children receiving home parenteral nutrition: a 20 year single center experience in 302 patients.
J Pediatr Gastroenterol Nutr 2007; 44(3):347-53.

21. Gandullia P, Lugani F, Costabello L, et al; Long- term home parenteral nutrition in children with chronic intestinal failure: A 15 year experience at a singleItalian centre. Dig Liver Dis 2011; 43(1):28-33.

22. Lacaille F, Irtan S, Dupic L, et al. Twentyeight years of intestinal transplantation in Paris: experience of the oldest European center. Transpl Int 2017; 30(2):178-86.

23. Martínez A, Wales PW. Intestinal transplantation in children: current status. Pediatr Surg Int 2016; 32(6):529-40.

24. Colomb V, Dabbas M, Goulet O, Talbotec C, Corriol O, Ricour C. Prepuberal growth in children with long-term parenteral nutrition. Horm Res 2002; 58 (1):2-6.

25. Bechard L, Rothpletz P, Touger R, Duggan C, Mehta N. N Influence of Obesity on Clinical Outcomes in Hospitalized Children. JAMA Pediatr 2013;176(5):476-82.

26. Oliveira C, Nasr A, Brindle M, Wales PW. Ethanol locks to prevent catheter-related bloodstream infections in parenteral nutrition: a meta-analysis. Pediatrics 2012;129(2): 318-29.

27. Ministerio de Salud de Chile; Programa Nacional de Salud de la Infancia con Enfoque Integral. En: Leyton B, Becerra C, Castillo C, Strain H, Santander S, Editores. Santiago, Chile: Editorial Valente; 2013. p. 84-6. 